\title{
Design, Preparation, and Evaluation of Enteric Coating Formulation of HPMC and Eudragit L100 on Carboxylated Agarose Hydrogel by Using Drug Tartrazine
}

\author{
Muhammad Junaid Khan, ${ }^{1}$ Wen-Can Huang, ${ }^{1}$ Muhammad Akhlaq, ${ }^{2}$ Sajid Raza, ${ }^{2}$ \\ Alkassoumi Hassane Hamadou, ${ }^{1}$ Guo Yuning, ${ }^{1}$ Jianan Sun $\mathbb{}^{1},{ }^{1}$ and Xiangzhao Mao ${ }^{1,3}$ \\ ${ }^{1}$ College of Food Science and Engineering, Ocean University of China, Qingdao 266003, China \\ ${ }^{2}$ Department of Pharmaceutics, Faculty of Pharmacy, Gomal University, Dera Ismail Khan 29050, Pakistan \\ ${ }^{3}$ Laboratory for Marine Drugs and Bioproducts of Qingdao National Laboratory for Marine Science and Technology, \\ Qingdao 266237, China
}

Correspondence should be addressed to Jianan Sun; sunjianan@ouc.edu.cn

Received 4 December 2021; Revised 28 December 2021; Accepted 15 January 2022; Published 28 January 2022

Academic Editor: Cleofe Palocci

Copyright (c) 2022 Muhammad Junaid Khan et al. This is an open access article distributed under the Creative Commons Attribution License, which permits unrestricted use, distribution, and reproduction in any medium, provided the original work is properly cited.

\begin{abstract}
Enteric-coated application on drug is used to prevent the drug from inactivation which are degraded by gastric enzyme. The present study is aimed at achieving controlled drug delivery in acidic medium of gastrointestinal tract (GIT) by enteric coating of hydroxy propyl methylcellulose (HPMC) and Eudragit L100 on carboxylated agarose hydrogel, creating a pH-dependent delivery system. Fourier-transformed infrared spectroscopy (FTIR) was for the detection of carboxylic group on agarose hydrogel, and scanning electron microscope (SEM) was used for the determination surface of prepared formulation. To check the $\mathrm{pH}$ sensitivity of enteric-coated formulation, different $\mathrm{pH}$ solution was used. It was found that the formulation was not dissolved in 1.2 but dissolve in pH 6.8 similarly; hydrogels lacking coating showed that tartrazine was more dissolved in $\mathrm{pH} 1.2$, and less dissolved at $\mathrm{pH}$ 6.8. The release of tartrazine from the hydrogels was measured by using spectrophotometer and using a scanning electron microscope to examine the morphology and surface appearance of hydrogel capsules. This study revealed cracks on coated samples, while noncoated samples showed clear appearance with no cracks. Our findings revealed that this method could be useful for the development of an enteric coating drug delivery system.
\end{abstract}

\section{Introduction}

Controlled drug delivery is intended to provide not only continued action but also dependability, which ideally means a zero-order release rate in which the amount of drug release to the absorption site remains reasonably constant over prolong period of time [1]. This system has been used at different interval of times to deliver the drugs at different predetermined rates in order to overcome the shortcomings of ordinary drug formulations [2].

For responsive drug release in various parts of the gastrointestinal tract (GIT), the $\mathrm{pH}$ range of fluids may provide the environmental catalyst [3]. When specific rate control is of secondary importance, the gels can be a useful agent for
$\mathrm{pH}$-triggered release. The inclination of $\mathrm{pH}$ in the GIT increased constantly from the stomach ( $\mathrm{pH} 1.2-3.5)$, followed by $\mathrm{pH}$ 5.7-6.8 along with the small intestine and colon (6.4-7.0), respectively [4]. Derivatives of cellulose and acrylic acid are commonly used as $\mathrm{pH}$-sensitive polymers. The delivery system needs to be designed based on the function properties of the polymers along with their solubility property at different $\mathrm{pH}$ environments in order to deliver the drugs at the target sites [5].

In addition, the field of controlled drug delivery requires further development of tools for the delivery of protein and peptide drugs. In the body, the appearance of a large number of biologically active peptides is strictly controlled to maintain normal metabolic stability through a reaction called 


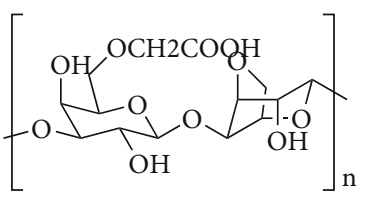

Carboxylated agarose

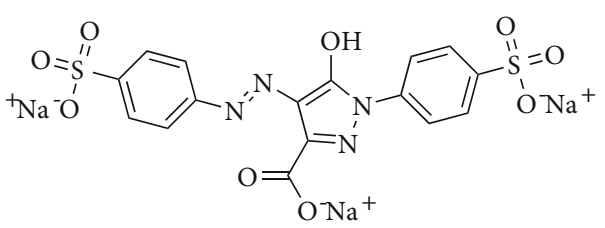

Tartrazine (i) $0.1 \mathrm{M} \mathrm{CaCl}_{2}$

(ii) $80^{\circ} \mathrm{C}, 10 \mathrm{~min}$

(iii) $4{ }^{\circ} \mathrm{C}$

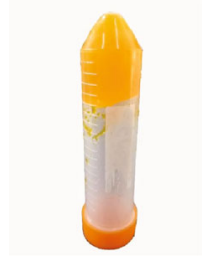

CTC Hydrogels

FIgURE 1: Synthesis of pH-sensitive hydrogels CTC.

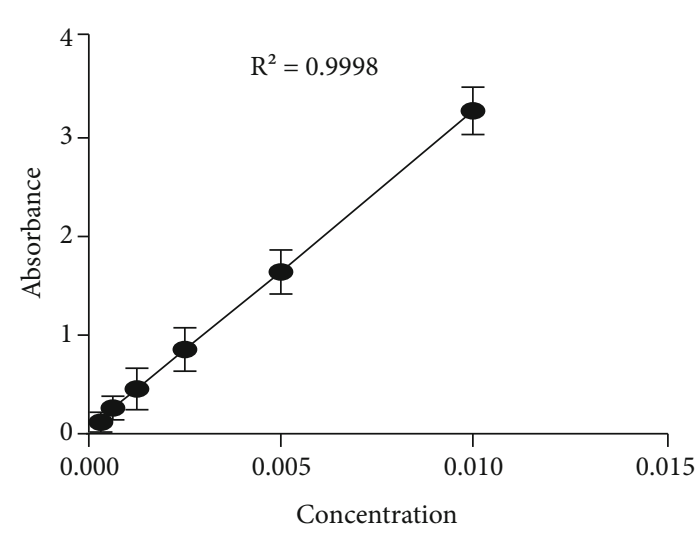

FIgURE 2: Tartrazine standard curve.

"homeostasis" [6]. If the active agent is delivered by a system that senses the signal caused by the disease, determines the signal strength, and, then takes action to release the appropriate amount of drugs, it will be very beneficial. Such a system would need to couple the drug delivery rate with physiological needs through some kind of feedback mechanism [7].

The $\mathrm{pH}$ range of fluids in different parts of the gastrointestinal tract (GIT) delivers environmental incentives for responsive drug release. [8] conducted studies on polymers containing weakly basic or acidic groups in the polymer backbone. The charge density of the polymer is contingent on the ion composition and $\mathrm{pH}$ value of the surface solution. Changing the $\mathrm{pH}$ of the solution will cause the polymer to swelling or deswelling. Therefore, the drug release of devices made of these polymers will show a release rate that is sensitive to $\mathrm{pH}$. [9] found that the swelling characteristics of multielement gels are also affected by the composition of the buffer. A practical result suggested is that these gels may not be able to dependably facilitate $\mathrm{pH}$-sensitive swelling controlled release in oral applications, because in the stomach, the level of buffer in the stomach is usually uncontrollable. [10] reported that more than two phases (collapse and swelling) can be found in gels composed of randomly distributed copolymers with negatively and positively charged groups. In these gels, the polymer segments interrelate through repulsion or attraction electrostatically and through hydrogen bonding and through repulsion or attraction electrostatic interaction.

Drug encapsulation/embedded hydrogels have different release mechanisms, such as swelling control, diffusion control, and chemical control mechanisms. The most acceptable mechanism is diffusion-controlled mechanism, and its drug



FIgURE 3: Tartrazine release rate of capsule-coated hydrogel in different $\mathrm{pH}$ mediums at various time intervals.

release follows Fick's law of diffusion. If the molecular size of the drug molecule is much smaller than the pore size of the porous hydrogel, the porosity of the hydrogel is related to the diffusion constant of the hydrogel. When the pore size in the hydrogel is equal to the size of the drug molecule, the release of the drug molecule is delayed by the cross-linked polymer chain. As a result, the diffusion constant is reduced. If the drug release rate surpasses the swelling rate, the drug release follows the swelling control mechanism [11].

The biodegradable polymers were first used by Heller and Trescony (1979) [12]. The controlled release designs used for oral administration have been changed by $\mathrm{pH}$ sensitive hydrogels. The stomach $\mathrm{pH}$ is less than 3 , which is quite different from the rather neutral $\mathrm{pH}$ of intestine [13]. This $\mathrm{pH}$ difference is sufficient for the development of $\mathrm{pH}$-sensitive polyelectrolyte hydrogels. For polycationic hydrogels, the swelling is negligible at neutral $\mathrm{pH}$, and it reduces the drug release from hydrogels. This property is useful for preventing the release of foul-tasting drugs in the neutral $\mathrm{pH}$ environment of the mouth [14]. When tartrazine was loaded into hydrogels made of copolymers of $\mathrm{N}, \mathrm{N}$ dimethylaminoethylmethacrylate (DMAEM) and methyl methacrylate, it was not released at neutral $\mathrm{pH}$. It can only be released at zero-order at $\mathrm{pH} 3-5$, where DMAEM was converted into ionized form. Various types of medications include tartrazine to give a yellow, orange, or green hue to a liquid, capsule, pill, lotion, or gel, primarily for easy identification [15]. 


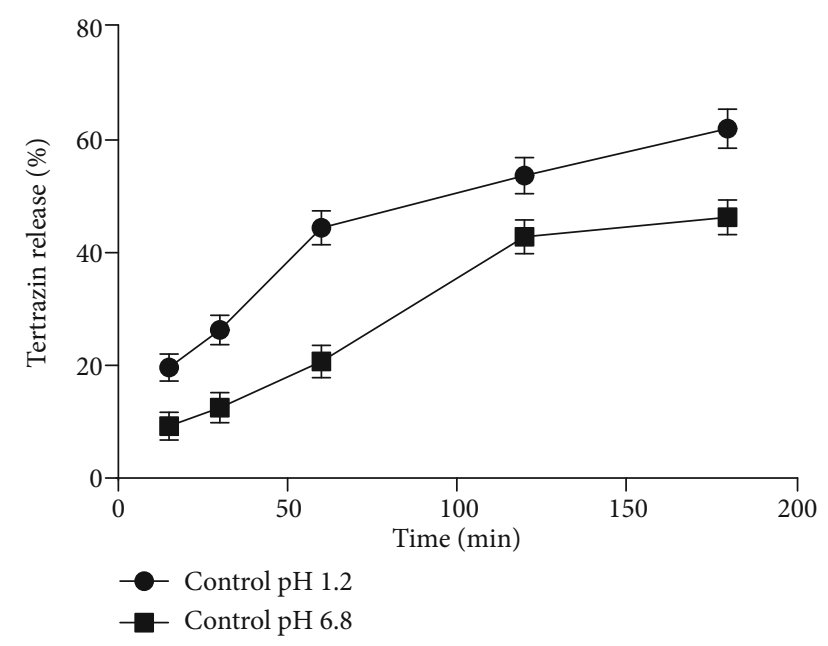

Figure 4: Tartrazine release rate of control hydrogels in different $\mathrm{pH}$ mediums at various time intervals.

Agarose is a natural polysaccharide that consists of 1, 3linked- $\beta$-d-galactose and 1, 4-linked 3, 6-anhydro $\alpha$-l-galactose with repeating units. Agarose has been broadly used in the biomedical field, such as cell biology [16], molecular biology [17], tissue engineering [18], and drug delivery [19]. They possess low resistance to shearing, heat, and acid, and they lack active signals to animate important cell processes [20], and these characteristics have limited their functional applications. Therefore, the properties of agarose have been modified by physical, chemical, and enzymatic processes or by combinations of them.

Enteric coatings are $\mathrm{pH}$-dependent, and therefore, have been used to stop gastric irritation or delay the release of drugs that are inactivated by the gastric acid in the stomach. In addition, such coatings may be applied to facilitate the delivery of a drug to its optimal absorption site in the intestine, thereby providing a delayed action, or for delivering the drug to its local site of action in the intestine [21].

Little information can be found in the literature regarding drug delivery systems and $\mathrm{pH}$-sensitive drug delivery mechanisms. Considering this research gap, the present work was designed with the following objectives: (1) to test the effect of coating on carboxylated agarose hydrogel and (2) to monitor the release of tartrazine in acidic $\mathrm{pH}$ medium.

\section{Materials and Methods}

2.1. Materials. Talc $\left(\mathrm{Mgso}_{3}\right)$, isopropanol, disodium hydrogen phosphate dodecahydrate $\left(\mathrm{H}_{25} \mathrm{Na}_{2} \mathrm{O}_{16} \mathrm{P}\right)$, sodium borohydride $\left(\mathrm{NaBH}_{4}\right)$, sodium hydrogen phosphate dihydrate (Na2HPO4), sodium bromide $(\mathrm{NaBr})$, and sodium chloride reagents were purchased from Sino Pharm Chemical Reagent Co. Ltd. (PR China). PEG 4000 was purchased from Solarbio, Shanghai, China, (2,2,6,6-Tetramethylpiperidin-1yl)oxyl TEMPO was purchased from Damas-beta, Shanghai, China, sodium hypochlorite $(\mathrm{NaOCl})$ was purchased from MACKLIN, Shanghai, China, and hydroxy propyl methylcellulose (HPMC) and Eudraget L 100 were purchased from Shanghai Dxiang Pharmaceutical Technology Co. Ltd.

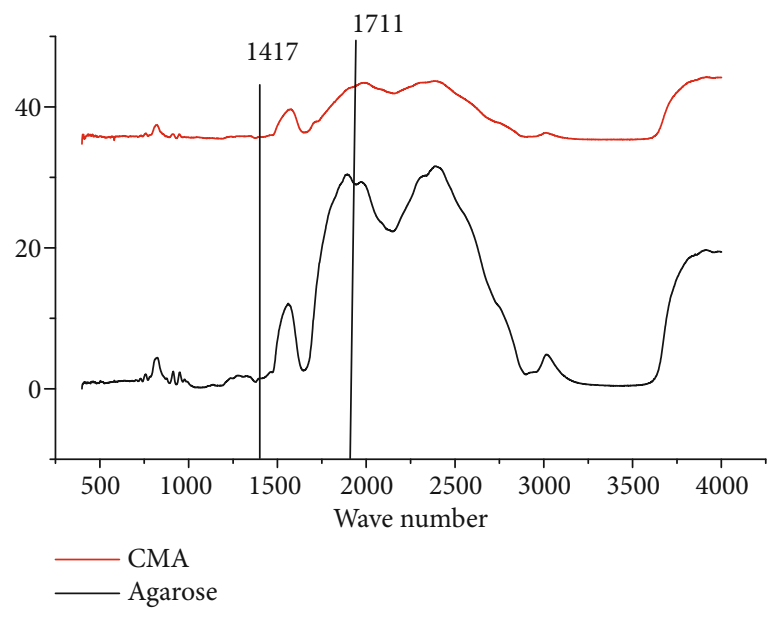

FIGURE 5: FTIR spectra of agarose and carboxylated agarose.

2.2. Synthesis of Carboxylated Agarose. Carboxylated agarose was prepared using the protocols of [22] with slight modifications. Briefly, a $50 \mathrm{~mL}$ methanol solution and $2 \mathrm{~g}$ of native agarose (NA) were dissolved into $100 \mathrm{~mL}$ of beaker using a stirrer, and $\mathrm{pH}$ was maintained using a $\mathrm{pH}$ meter. The solution was heated to $80^{\circ} \mathrm{C}$ to dissolve agarose and then chilled at $0^{\circ} \mathrm{C}$ under continuous stirring to avoid gel formation of the solution. When the solution was cooled, TEMPO (0.160 mmol, $1.14 \mathrm{mmol}), \mathrm{NaBr}(0.9 \mathrm{mmol}, 0.02 \mathrm{mmol})$, and $\mathrm{NaOCl}(2.5 \mathrm{~mL}, 15 \% \mathrm{vol} / \mathrm{vol}$ solution) were added under constant stirring. The solution was kept at a $\mathrm{pH}$ of 10.8 until the end of the reaction, and $\mathrm{NaOH}$ solution $(0.5 \mathrm{M})$ was used to control the $\mathrm{pH}$. At the end of the reaction, $\mathrm{NaBH}_{4}(0.1 \mathrm{~g})$ was added, and the solution was acidified to $\mathrm{pH} 8$ and stirred for $1 \mathrm{~h}$. To precipitate carboxylated agarose (CA), $\mathrm{NaCl}$ $(0.2 \mathrm{~mol} / \mathrm{L}, 12 \mathrm{~g})$ and ethanol $(500 \mathrm{~mL})$ were sequentially added. The solution was then collected using vacuum filtration and extracted by ethanol. The remaining ethanol was removed by extensive dialysis against water, and the CA was achieved as a white solid upon freeze-drying overnight. Carboxylation was confirmed using Fourier-transformed infrared spectra (FTIR) analysis.

2.3. Preparation of Hydrogels. CA $(2 \% w / v)$ was dissolved in distilled water $(15 \mathrm{~mL})$ at $80^{\circ} \mathrm{C}$, and $0.03 \mathrm{~g}$ tartrazine was added to provide a final tartrazine concentration of $0.5 \% \mathrm{w}$ $/ v$. Following that, $0.1 \mathrm{M} \mathrm{CaCl}_{2}(1 \mathrm{~mL})$ was added to this solution and stirred for 20 minutes. Calcium ion was used for cross-linking on the $\mathrm{pH}$ release of tartrazine to be investigated. CA was designated for this study as it provides hydrogel with desired mechanical properties, where assailow tartrazine and $\mathrm{CaCl}_{2}$ concentrations were chosen to decrease the potential side effects. The present study utilized $\mathrm{Ca}^{2+}$ ions, as they provided the best control over the release of tartrazine [22].

2.4. Preparation of Precoating and Overcoating Solution. Coatings on the hydrogel capsules frequently undergo insufficient bonding between the shell and the coating. Thus, previous researchers in the field of enteric coating have found that it is needed to precoat hydrogel capsules [23]. We used 




(a)

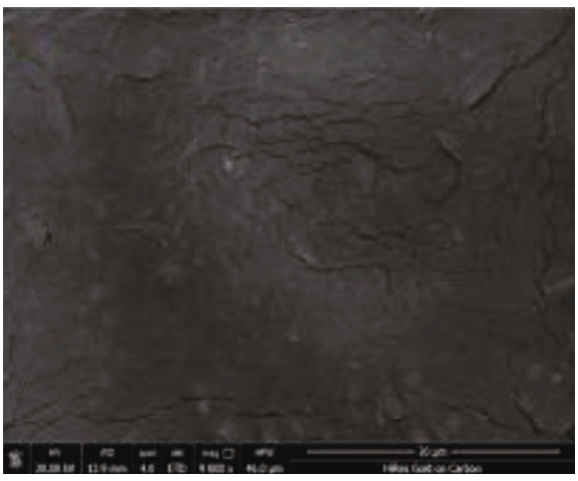

(c)

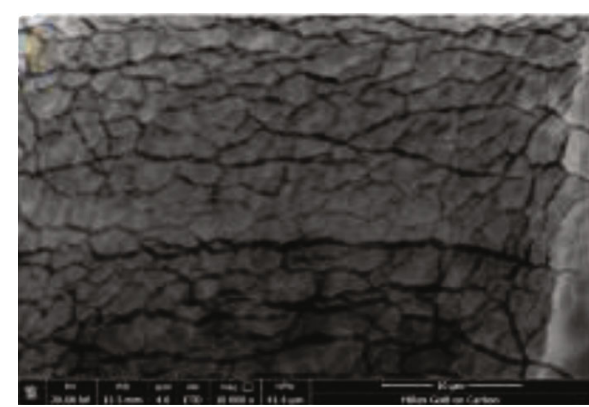

(b)



(d)

FIGURE 6: SEM image of coating ( $a, b)$ and noncoating (c, d) hydrogels.

three different coating stages: precoating, protective coating or enteric coating, and overcoating. One accurately weighed gram of HPMC was dissolved in two-thirds of mixed solvents of ethanol and methylene chloride $20 \mathrm{~mL}$ (1:1 ratio) with stirring and was mixed for 30 minutes. Finally, the remaining solution was added into the mixture under constant stirring until the solution was clearly soluble. A similar process was followed for over coating solution.

2.5. Preparation of Enteric Coating Solution. Eudragit L 100 of $3 \mathrm{~g}$ was dissolved in $40 \mathrm{~mL}$ of isopropanol and acetone ( $1: 1)$ by using magnetic stirrer. Then, PEG 4000 (14\% based on the film former) and talc (6.0\%) were added into the above solution with continuous stirring until the solution was fully dissolved.

2.6. Preparation of Eudragit L 100-Coated Capsules. CA of $0.3 \mathrm{~g}$ was dissolved in $15 \mathrm{~mL}$ distilled water at $90^{\circ} \mathrm{C}$. When the CA was fully dissolved, the hydrogel solution was transferred into an ellipsoid mold to make hydrogel capsules. Uncoated capsules were placed in a Petri dish and were coated by syringe spraying. Initially, precoating solution (HPMC) was used for $30 \mathrm{~min}$, and the temperature was maintained between 30 and $35^{\circ} \mathrm{C}$. Next, the enteric coating solution (Eudragit L 100) was sprayed.

2.7. Fourier-Transformed Infrared Spectroscopy (FTIR). Sample (1 mg) and $\mathrm{KBr}(100 \mathrm{mg})$ were crushed using a grinder and mortar and then pressed for 5 min into discs by using a 10-ton press. Fourier-transformed infrared spectroscopy (FTIR) spectra were recorded on a Vector 22 instrument
(Bruker Optics), and the software provided by the manufacturer was used to analyze and import the spectrum.

2.8. Scanning Electron Microscope (SEM). The morphology and surface appearance of hydrogel capsules and of coating and noncoating hydrogel capsules were examined with a scanning electron microscope (FEI Quanta FEG 650) equipped with an image analysis system. Prior to examination, the samples were gold sputter-coated to make them electrically conductive.

2.9. In Vitro Release Studies. Tartrazine $100 \mathrm{~mL}$ stock arrangement was set up by dissolving $10 \mathrm{mg}$ of it in $100 \mathrm{~mL}$ of volumetric flask by utilizing phosphate buffer $\mathrm{pH}$ (6.8), and to make the volume upto $100 \mathrm{~mL}$, more phosphate buffer was in added. The stock solution contains $0.1 \mathrm{mg} / \mathrm{mL}$ of tartrazine. In tartrazine stock solution, $50 \mathrm{~mL}$ solution of it was diluted in $100 \mathrm{~mL}$ volumetric flask by adding $50 \mathrm{~mL}$ of phosphate buffer $(6.8 \mathrm{pH})$. The dilutions made from the stock solution contain $0.05 \mathrm{mg}$ of tartyrazine, and $50 \mathrm{~mL}$ solution was taken in $100 \mathrm{~mL}$ volumetric flask, and to make the volume upto $100 \mathrm{~mL}$, more phosphate buffer of $(6.8 \mathrm{pH})$ were added. Each $\mathrm{mL}$ contains $0.025 \mathrm{mg}$ of tartrazine. Similarly, dilutions of $0.0125 \mathrm{mg} / \mathrm{mL}, 0.00625 \mathrm{mg} /$ $\mathrm{mL}$, and $0.003125 \mathrm{mg} / \mathrm{mL}$ of tartrazine were prepared. Spectrophotometer (BioTek, PowerWave XS2, VT, USA) was used for analysis of tartrazine in dilute samples at $426 \mathrm{~nm}$.

The release of tartrazine from the hydrogels was measured spectrophotometrically using a microplate spectrophotometer (BioTek, PowerWave XS2, VT, USA). The hydrogel precursor mixture $(100 \mu \mathrm{L})$ was added to the 
bottom of the 96 well plate and set at room temperature before the addition of medium $(4 \mathrm{~mL})$ at $\mathrm{pH}$ of 1.2 and 6.8 . The plate was transferred to a UV spectrophotometer and set to $37^{\circ} \mathrm{C}$. Time-based measurements of tartrazine release were conducted over 15, 30, 60, 120, and $180 \mathrm{~min}$. The tartrazine released into the medium was determined by measuring its absorbance at $426 \mathrm{~nm}$ in comparison to the standard curve. The release curve was plotted as the percentage of drug released versus time.

\section{Results and Discussion}

3.1. pH-Responsive Hydrogel Fabrication. pH-responsive hydrogels were prepared from carboxylated agarose, tartrazine, and $\mathrm{CaCl}_{2}$ and were referred to as carboxylated tartrazine calcium chloride (CTC) hydrogels (Figure 1). The CA is a derivative of agarose, which is the primary hydrogel group of the galactose units oxidized to carboxylic acid, and CA was selected for this study because of its good capability to form $\mathrm{pH}$-responsive ionic interaction. Tartrazine was chosen to increase the rate of pigments. The hydrogels were prepared by dissolving CA in water at $80^{\circ} \mathrm{C}$ by adding tartrazine and $\mathrm{CaCl}_{2}$ to make hydrogel precursor solution and then cooled to $4^{\circ} \mathrm{C}$ to make hydrogels (Figure 1). Calcium ions were used as a cross linker to make the ionic bond between carboxylated agarose and tartrazine.

3.2. Enteric Coating with Release Rate. Standard curve was prepared by dissolving in tartrazine in phosphate buffer with pH 7.4 and was constructed by plotting absorbance vs. concentration, and the corresponding $R^{2}$ value is .9998 as shown Figure 2. The present study results showed that coated hydrogel capsules were not well dissolved in $\mathrm{pH} 1.2$, but were well dissolved at pH6.8 (Figure 3). Similarly, noncoated hydrogels showed that tartrazine is more easily dissolved at pH 1.2 and less dissolved at $\mathrm{pH}=6.8$ (Figure 4). Other studies also indicated that an enteric coating could be a polymer abstraction connected in oral medicine, enabling it to protect the drug from acidic $\mathrm{pH}$ environments, e.g., the stomach [24]. [25] also reported that the enteric or nonenteric hydrogels are the solutions having some other ingredients that may facilitate the application of the fabric coating. Enteric coating works with high efficiency when it is present at the surface of a highly acidic environment, such as the stomach, but breaks down quickly at a less acidic pH. For example enteric coating solution will not break up at the $\mathrm{pH}$ of stomach acid (1.2) but will break up in the alkaline $\mathrm{pH}$ (6.8) environment present in the small intestine. The dissolution of tartrazine on a coating capsule was used to evaluate the pigments on the release of tartrazine. The phosphate buffer solution is used for the dissolution experiments. $\mathrm{pH}$ (1.2) was used as the accelerated gastric fluid, and $\mathrm{pH}$ (6.8) was used as the small intestinal fluid. Initially, phosphate buffer solution was made and set in two different $\mathrm{pHs:} \mathrm{pH}(1.2)$ and $\mathrm{pH}$ (6.8). Then, a hydrogel capsule was made, and it was coated with a Eudragit L 100 solution and HPMC.

Furthermore, HPMC was coated with hydrogel and kept for 1 hour at $35^{\circ} \mathrm{C}$. Following that, HPMC coated again with Eudragit L 100 solution via syringe spraying. During the experiment for tartrazine release by using a microplate spectrophotometer (Bio Tek, Power Wave XS2, VT, USA), readings were recorded at various time intervals $(15,30,60,120$, and $180 \mathrm{~min}$ ).

3.3. Fourier-Transformed Infrared Spectroscopy (FTIR). FTIR spectra and modified carboxylated agarose are shown in Figure 5. The agarose was compared with carboxylated agarose. The appearance of new peaks in the spectrum of the carboxylated agarose at $1417 \mathrm{~cm}^{-1}$ and $1711 \mathrm{~cm}^{-1}$ might be applied to -COO- symmetric strain and antisymmetric strain. The strains showed that there were carboxylated group in agarose chains after oxidation of TEMPO.

3.4. Scanning Electron Microscope (SEM). Analysis of surface morphology was performed by scanning electron microscope with different media. The hydrogel and Eudragit L100 coating on the capsule were successfully formed. The samples that were coated developed cracks as shown in (Figures 6(a) and 6(b)). On the other hand, the samples that were not coated show no cracks and had clear appearance as shown in (Figures 6(c) and 6(d)). These results were similar to the result of [26], who found that the surface of the capsule was relatively smooth. Thus, the present results show that a good coating surface layer has smooth surface while the capsule having no surface layer represent rough surface.

\section{Conclusion}

Controlled drug delivery system is effective and most appropriate method for efficient delivery of drugs in acidic medium in GIT. In our study, tartrazine was released in stomach and intestine fluids on the acidic $\mathrm{pH}$ medium. This study showed the effective release of the drug in the respective targeted medium. However, on the basis of these results, it is suggested that further studies should be conducted regarding controlled drug delivery systems to strengthen the information provided in this work. Hydrogel was used as a controlled release polymer for tartrazine. FTIR were used for interaction study and SEM for coting surface. From the results of test the pHs of 1.2 and 6.8 for tartrazine release from the hydrogel, it was found that the release was appropriate at $6.8 \mathrm{pH}$.

\section{Data Availability}

The data used to support the findings of this study are included within the article.

\section{Additional Points}

Highlights. (i) Enteric-coated application on tartrazine. (ii) The drug delivery system to prevent the drug from gastric HCL. (iii) Tartrazine release was minimum at $\mathrm{pH} 1.2$ and maximum at $\mathrm{pH} 6.8$.

\section{Conflicts of Interest}

The authors declare that they have no conflicts of interest. 


\section{Acknowledgments}

This work was supported by the National Natural Science Foundation of China (31922072), Taishan Scholar Project of Shandong Province (tsqn201812020), and Fundamental Research Funds for the Central Universities (201941002).

\section{References}

[1] S. M. Dadou, M. D. Antonijevic, B. Z. Chowdhry, and A. A. Badwan, An Overview of Chitosan-Xanthan Gum Matrices as Controlled Release Drug Carriers, IntechOpen, 2018.

[2] A. Gangrade and B. B. Mandal, "Injectable carbon nanotube impregnated silk based multifunctional hydrogel for localized targeted and on-demand anticancer drug delivery," ACS Biomaterials Science \& Engineering, vol. 5, no. 5, pp. 2365-2381, 2019.

[3] T. Tarvainen, T. Nevalainen, A. Sundell et al., "Drug release from poly(acrylic acid) grafted poly(vinylidene fluoride) membrane bags in the gastrointestinal tract in the rat and dog," Journal of Controlled Release, vol. 66, no. 1, pp. 19-26, 2000.

[4] D. S. Seeli and M. Prabaharan, "Guar gum oleate-graft-poly(methacrylic acid) hydrogel as a colon-specific controlled drug delivery carrier," Carbohydrate Polymers, vol. 158, pp. 51-57, 2017.

[5] V. Balamuralidhara, T. M. Pramodkumar, N. Srujana et al., "pH sensitive drug delivery systems: a review," American Journal of Drug Discovery and Development, vol. 1, no. 1, pp. 2448,2011

[6] C. J. Pérez-Martínez, S. D. Morales Chávez, T. del CastilloCastro et al., "Electroconductive nanocomposite hydrogel for pulsatile drug release," Reactive and Functional Polymers, vol. 100, pp. 12-17, 2016.

[7] J. R. K. Reddy, M. V. Jyothsna, T. M. Saleem, and C. M. S. Chetty, "Review on: pulsatile drug delivery systems," Journal of Pharmaceutical Sciences and Research, vol. 1, no. 4, p. 109, 2009.

[8] S. Juodkazis, N. Mukai, R. Wakaki, A. Yamaguchi, S. Matsuo, and H. Misawa, "Reversible phase transitions in polymer gels induced by radiation forces," Nature, vol. 408, no. 6809, pp. 178-181, 2000.

[9] A. Serrano-Medina and J. M. Cornejo-Bravo, "Buffer effects on drug release kinetics from acidic hydrophobic gel discs," Journal of the Mexican Chemical Society, vol. 55, no. 1, pp. 2-6, 2011.

[10] P. Gupta, K. Vermani, and S. Garg, "Hydrogels: from controlled release to $\mathrm{pH}$-responsive drug delivery," Drug Discovery Today, vol. 7, no. 10, pp. 569-579, 2002.

[11] C. C. Lin and A. T. Metters, "Hydrogels in controlled release formulations: network design and mathematical modeling," Advanced Drug Delivery Reviews, vol. 58, no. 12-13, pp. 1379-1408, 2006.

[12] J. Heller and P. Trescgny, "Controlled drug release by polymer dissolution II: enzyme-mediated delivery device," Journal of Pharmaceutical Sciences, vol. 68, no. 7, pp. 919-921, 1979.

[13] B. E. F. de Ávila, P. Angsantikul, J. Li et al., "Micromotorenabled active drug delivery for in vivo treatment of stomach infection," Nature Communications, vol. 8, no. 1, pp. 1-9, 2017.

[14] K. Dutta, R. Das, J. Ling et al., "In situ forming injectable thermoresponsive hydrogels for controlled delivery of biomacromolecules," ACS Omega, vol. 5, no. 28, pp. 17531-17542, 2020.
[15] A. Arbe, F. Alvarez, and J. Colmenero, "Insight into the structure and dynamics of polymers by neutron scattering combined with atomistic molecular dynamics simulations," Polymers, vol. 12, no. 12, p. 3067, 2020.

[16] W. Yi and Q. OuYang, "Adiponectin improves diabetic nephropathy by inhibiting necrotic apoptosis," Archives of Medical Science, vol. 15, no. 5, pp. 1321-1328, 2019.

[17] S. Saeedi Garakani, M. Khanmohammadi, Z. Atoufi et al., "Fabrication of chitosan/agarose scaffolds containing extracellular matrix for tissue engineering applications," International Journal of Biological Macromolecules, vol. 143, pp. 533-545, 2020.

[18] Y. du, H. Liu, Q. Yang et al., "Selective laser sintering scaffold with hierarchical architecture and gradient composition for osteochondral repair in rabbits," Biomaterials, vol. 137, pp. 37-48, 2017.

[19] C. Kim, D. Jeong, S. Kim, Y. Kim, and S. Jung, "Cyclodextrin functionalized agarose gel with low gelling temperature for controlled drug delivery systems," Carbohydrate Polymers, vol. 222, article 115011, 2019.

[20] S. Yixue, C. Bin, G. Yuan et al., "Modification of agarose with carboxylation and grafting dopamine for promotion of its cell-adhesiveness," Journal of Carbohydrate Polymers, vol. 92, no. 2, pp. 2245-2251, 2013.

[21] P. K. Pawar and C. Gautam, "Design, optimization and evaluation of mesalamine matrix tablet for colon drug delivery system," Journal of Pharmaceutical Investigation, vol. 46, no. 1, p. $6778,2015$.

[22] N. Ninan, A. Forget, V. P. Shastri, N. H. Voelcker, and A. Blencowe, "Antibacterial and anti-inflammatory pHresponsive tannic acid-carboxylated agarose composite hydrogels for wound healing," ACS Applied Materials \& Interfaces, vol. 8, no. 42, pp. 28511-28521, 2016.

[23] E. T. Cole, R. A. Scott, A. L. Connor et al., "Enteric coated HPMC capsules designed to achieve intestinal targeting," International Journal of Pharmaceutics, vol. 231, no. 1, pp. 83-95, 2002.

[24] B. D. Ratner, Biomaterials Science: An Introduction to Materials in Medicine, Elsevier Academic Press, San Diego, Calif, 2nd edition, 2004.

[25] S. Islam, F. Khatun, A. Bakr, H. Ibrahim Mondal, M. Haque, and A. Mahmud, "A review on biodegradable polymers for enteric coating," Human Journals, vol. 6, no. 3, pp. 141-159, 2016.

[26] F. Muhammed, S. Pollington, X. Sun, A. Abdullah, and Y. Liu, "Novel coatings on zirconia for improved bonding with veneer ceramics," Coatings, vol. 8, no. 10, p. 363, 2018. 